\title{
One-and-a-half-centered expansion method in charge-transfer calculations of proton-hydrogen scattering
}

\author{
Zhifan Chen* and J. F. Reading \\ Center for Theoretical Physics, Department of Physics, Texas A\&M University, College Station, Texas 77843
}

(Received 13 May 1992; revised manuscript received 1 March 1993)

\begin{abstract}
In this paper, we undertake a feasibility study of improving the one-and-a-half-centered expansion (OHCE) method of Reading, Ford, and Becker [J. Phys. B 14, 1995 (1981); 15, 3257 (1982)]. We have explored the efficacy of an alternative method to evaluate the charge-transfer matrix elements and improved the estimated time dependence of the charge-transfer scattering amplitudes. More projectile states have been included in the calculations than used hitherto. A unitary matrix, $U$ matrix, which can propagate the wave functions from $-\infty$ to $t$, where $t$ denotes time, has been constructed using the single-centered expansion (SCE) method. A complex basis set of nine radial $s$ states and nine radial $p$ states has been used in the expansion of trial wave functions for the target. Charge-transfer matrix elements have been evaluated by a Feynman integral technique; one numerical integral using Gaussian quadrature is needed. The radial parts of the matrix elements are stored on circles and used for all the impact parameters. In a OHCE calculation, we have to choose a function $\beta_{m}(z)$ to modulate the charge-transfer amplitudes. The only constraints on $\beta_{m}(z)$ are $\beta_{m}(-\infty)=0$ and $\beta_{m}(\infty)=1$. In this paper, $\beta_{m}(z)$ has been obtained from a SCE calculation. This $\beta_{m}(z)$ function increases gradually in the whole collision region. It offers an improvement over the step function used in previous work. A computer code has been developed to include $s$ and $p$ states for the target and projectile. The calculations have been performed in the proton energy range from 30 to $250 \mathrm{keV}$. The charge transfer to the $1 s$ state has been calculated and gives good agreement with the experimental data. The proton energy ranges have been extended from the $100 \mathrm{keV}$ used in previous work to $250 \mathrm{keV}$. The charge-transfer cross sections to the $2 p$ state fit the experimental data at $30 \mathrm{keV}$ and are almost the same as those calculated using the four-state, two-centered expansion method proposed by Cheshire and Gallaher [J. Phys. B 3, 813 (1970)] and Shakeshaft [Phys. Rev. A 14, 1626 (1976)]. The results of the charge exchange to the $2 s$ state are also in fairly good agreement with the measurements of Ryding [listed in Tawara, Kato, and Nakar, At. Data Nucl. Data Tables 32, 235 (1985)].
\end{abstract}

PACS number(s): 34.70. +e, 82.30.Fi, 34.50. - s, 34.80.-i

\section{INTRODUCTION}

Electron-capture processes in ion-atom collisions play important roles in astrophysics, atmospheric physics, and plasma physics. For many years, charge transfer in the reactions of protons and hydrogen atoms has received a great deal of attention, both theoretical and experimental. Several theoretical methods such as Born series [1-4], distortion approximation [5,6], impulse approximation [7-9], molecular-state expansion [10-12], and atomicstate expansion [13-21] have been used to evaluate the cross sections of electron exchange. A two-state atomicexpansion method was invented by Bates [22]. His theory has been applied in the symmetrical resonance process [13] and nonresonance processes [6,14]. Bates and Boyd [20] have shown that except at very low energies, the relative velocity vector of the colliding systems can be assumed to be constant. For proton-hydrogen collisions the assumption is valid down to a $100 \mathrm{eV}$ of proton energies. The problem is thus simplified to that of finding the solutions of the time-dependent Schrödinger equation for an electron with appropriate initial conditions.

McElroy [6] calculated charge-exchange cross sections for $1 s-2 s, 1 s-2 p$ in proton-hydrogen collisions using a dis- tortion approximation. McCarroll [13] and Lovell and McElroy [14] did calculations of $1 s-1 s$ and $1 s-2 s$ charge transfer using two-center, two-state expansions. Wilets and Gallaher [15] performed coupled-channel calculations with $1 s, 2 s$, and $2 p$ states centered around either proton. The energy range covered by them was from 1 to $100 \mathrm{keV}$. The charge-transfer matrix elements were evaluated by two-dimensional numerical integral Cheshire and Gallaher [16] calculated the same reaction in the energy range from 1 to $1000 \mathrm{keV}$. They used a differentialequation method [21] to determine the matrix elements. Rapp, Dinwiddie, and Storm [17,18] and Shakeshaft [19] also performed close-coupling calculations. The results of Shakeshaft and Cheshire and Gallaher more or less agree with each other, but are somewhat different from those obtained by Wilets and Gallaher. The discrepancy may be attributed to the inaccuracy in numerical calculation. These two-centered expansions (TCE) consume much computing time.

Reading, Ford, and Becker [23-25] have invented a set of one-and-a-half-centered expansion (OHCE) methods. These methods allow a shorter computational time and systematic improvement in its accuracy. However, previous OHCE calculations were restricted to only the $1 s$ electron state on the projectile and an unphysical step 
function was used to modulate the charge-transfer amplitudes. The charge-transfer matrix elements were evaluated by a partial-wave expansion method [23]. This method is expected to be inaccurate at high energies. In this paper we have explored the efficacy of an alternative method to evaluate the charge-transfer matrix elements and improved the estimated time dependence of the charge-transfer scattering amplitudes of proton-hydrogen scattering in OHCE calculations. We allow $s$ and $p$ states for the projectile. As limited by the memory space most of the calculations are performed with bases that include four states $\left(1 s, 2 s, 2 p_{0}, 2 p_{1}\right)$ on the projectile and nine radial $s$ states $(2 s$ to $9 s)$ and nine radial $p$ states $\left(2 p_{0}\right.$ to $10 p_{0}$ and $2 p_{1}$ to $10 p_{1}$ ) for the target. The charge transfer to the $1 s$ states has been calculated and gives good agreement with the experimental data. The proton energy ranges have been extended from $100 \mathrm{keV}$ in previous work to $250 \mathrm{keV}$. The charge-transfer cross sections to the $2 p$ state fit the experimental data at $30 \mathrm{keV}$ and are almost the same as those calculated using TCE method by Cheshire and Shakeshaft in the energy ranges from $\mathbf{3 0}$ to $250 \mathrm{keV}$. Charge transfer to the $2 s$ state is also in fairly good agreement with Ryding's experimental data [27].

\section{THEORY AND NUMERICAL METHOD}

Consider a proton incident on a target hydrogen atom in which the nucleus is fixed at the origin of the coordinate system during the collision process. The coordinate system is described in Fig. 1. $\mathbf{R}$ and $\mathbf{r}$ are the position vector of the projectile (mass $M$ ) and target electron (mass $m$ ). In the semiclassical model a straight-line trajectory which is distinguished by an impact parameter $\mathbf{B}$ and a velocity $\mathbf{v}$ has been defined for the motion of the proton. The electronic motion is obtained by solving the time-dependent Schrödinger equation, in atomic units,

$$
i v \frac{\partial}{\partial z} \Psi=\left(-\frac{1}{2} \nabla_{r}^{2}+V_{p}+V_{t}\right) \Psi,
$$

where the time-varying potential arises through $\boldsymbol{R}(t)$.

The interactive potentials $V_{p}$ and $V_{t}$ include two terms. The second terms will guarantee that the potentials go to zero faster than just keeping the first term and make scattering amplitudes to be constants when $R \rightarrow \infty$

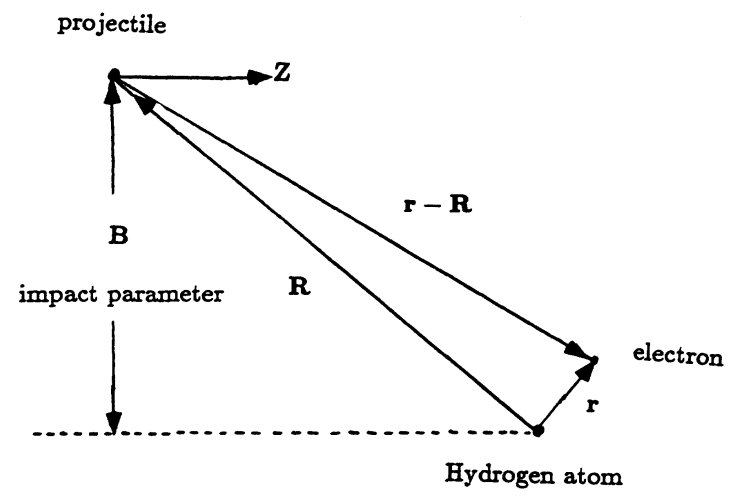

FIG. 1. Collision and coordinate systems.

$$
\begin{aligned}
& V_{p}=-\frac{1}{|\mathbf{r}-\mathbf{R}|}+\frac{1-e^{-R}}{R}, \\
& V_{t}=-\frac{1}{r}+\frac{1-e^{-R}}{R} .
\end{aligned}
$$

In the OHCE method,the trial wave functions are taken as

$$
\Psi(\mathrm{OHCE})=\sum_{n=1}^{N} a_{n}(z) \chi_{n}(\mathbf{r}, z)+\sum_{m=1}^{M} b_{m} \beta_{m}(z) \phi_{m}(\mathbf{r}, z),
$$

where $\chi_{n}(\mathbf{r}, z)$ and $\phi_{m}(\mathbf{r}, z)$ are the target wave functions and projectile wave functions, respectively. They can be obtained from the diagonalization of the Hamiltonian on a basis $\left[\left(Y_{l, m}+Y_{l, m}^{*}\right) / \sqrt{(2)}\right] r^{l} e^{-\alpha_{i} r} \quad(m \neq 0)$ and $Y_{l, 0} r^{l} e^{-\alpha_{i} r}$ otherwise. The $\alpha_{i}$ are listed:

$$
\begin{array}{ll}
\alpha_{1}=1.0000+0.0000 i, & \alpha_{2}=0.1708+0.0000 i, \\
\alpha_{3}=02476-0.1241 i, & \alpha_{4}=0.4041-0.3544 i, \\
\alpha_{5}=0.6751-0.6581 i, & \alpha_{6}=0.1328-0.8799 i, \\
\alpha_{7}=0.1328+0.8799 i, & \alpha_{8}=0.6751+0.6581 i, \\
\alpha_{9}=0.4041+0.3544 i, & \alpha_{10}=0.2476+0.1241 i .
\end{array}
$$

Details of such pseudostate expansion and diagonalization are stated in Ref. [26]. As an example the wave functions of $s$ states in Eq. (2.4) can be expressed by

$$
\begin{array}{r}
\chi_{n}(\mathbf{r}, z)=\sqrt{1 / 4 \pi} \sum_{i} g_{i s} e^{-\alpha_{i} r} e^{-i E_{i} Z / v} \\
\quad \times \exp \left[i \frac{1}{v} \int_{-\infty}^{z} \frac{1-e^{-R}}{R} d z^{\prime}\right], \\
\phi_{m}(\mathbf{r}, z)=\sqrt{1 / 4 \pi} \sum_{i} q_{i s} e^{-i E_{i} z / v} e^{-\alpha_{i}|\mathbf{r}-\mathbf{R}|} e^{i \mathbf{v} \cdot \mathbf{r}-i v Z / 2} \\
\times \exp \left[i \frac{1}{v} \int_{-\infty}^{z} \frac{1-e^{-R}}{R} d z^{\prime}\right],
\end{array}
$$

where $R=(Z, B)$ and $g_{i s}, q_{i s}$ are the coefficients from the diagonalization of the Hamiltonian of hydrogen atom. To account for the second term of $V_{P}$ and $V_{T}$, a phase factor $\exp \left\{i(1 / v) \int_{-\infty}^{z}\left[\left(1-e^{-R}\right) / R\right] d z^{\prime}\right\}$ has been introduced in the wave functions of the target and the projectile. By introducing a $z, x$, or $z-Z$, etc., the wave functions of the $p$ state can be expressed in a similar way. In Eq. (2.4) $a_{n}$ are the excitation amplitudes. The condition

$$
\left\langle\chi_{n}\left|i v \frac{\partial}{\partial z}-H\right| \Psi(\mathrm{OHCE})\right\rangle=0
$$

gives the $a_{n}$

$$
\begin{aligned}
a_{n}=c_{n}-\langle i / v\rangle \sum_{n^{\prime}, n^{\prime \prime}, m} U_{n n^{\prime}}(z,-\infty) \\
\times \int_{-\infty}^{+\infty} d z^{\prime} U_{n^{\prime} n^{\prime \prime}}^{\dagger}\left(z^{\prime},-\infty\right) \\
\times d_{n^{\prime \prime} m}\left(z^{\prime}\right) b_{m},
\end{aligned}
$$


where

$$
d_{n^{\prime \prime} m}(z)=\left\langle\chi_{n^{\prime \prime}}\left|V_{t}\right| \phi_{m}\right\rangle \beta_{m}(z)-\left\langle\chi_{n^{\prime \prime}} \mid \phi_{m}\right\rangle i v \frac{\partial \beta_{m}(z)}{\partial z} .
$$

The $b_{m}$ are the constants. The only constraints on the chosen functions $\beta_{m}(z)$ are the boundary conditions

$$
\beta_{m}(-\infty)=0, \beta_{m}(\infty)=1
$$

In our OHCE calculations the $\beta_{m}(z)$ are chosen as

$$
\beta_{m}(z)=\frac{\int_{-\infty}^{z} d z^{\prime}\left\langle\phi_{m}\left|V_{t}\right| \Psi(\mathrm{SCE})\right\rangle}{\int_{-\infty}^{+\infty} d z^{\prime}\left\langle\phi_{m}\left|V_{t}\right| \Psi(\mathrm{SCE})\right\rangle}
$$

therefore

$$
\frac{\partial \beta_{m}(z)}{\partial z}=\frac{\left\langle\phi_{m}\left|V_{t}\right| \Psi(\mathrm{SCE})\right\rangle}{\int_{-\infty}^{+\infty} d z^{\prime}\left\langle\phi_{m}\left|V_{t}\right| \Psi(\mathrm{SCE})\right\rangle} .
$$

This $\beta_{m}(z)$ function increases smoothly from 0 at $-\infty$ to 1 at $\infty$. It offers an improvement over the step function used in previous work. In OHCE $b_{m}$ and $\beta_{m}(z)$ are no longer the time-dependent variational parameters. In Eq. (2.8), $U_{n n^{\prime}}(z,-\infty)$ is a $U$ matrix in the single-centered expansion (SCE). The $U$ matrix, which propagates the wave function from $z_{0}$ to $z$, can be obtained by

$$
U_{n n^{\prime}}\left(z, z_{0}\right)=\exp \left[-\frac{i}{v} \int_{z_{0}}^{z} V_{n n^{\prime}}\left(z^{\prime}\right) d z^{\prime}\right],
$$

where $V_{n n^{\prime}}\left(z^{\prime}\right)$ is the excitation matrix element. The $c_{n}$ in Eq. (2.8) are the first column of $U$.

The perturbative constraint is

$$
\int_{-\infty}^{+\infty} d z\left\langle\phi_{m}\left|i v \frac{\partial}{\partial z}-H\right| \Psi(\mathrm{OHCE})\right\rangle=0 .
$$

Substituting $a_{n}$, Eqs. (2.9) and (2.10) into Eq. (2.12), we obtain the scattering amplitude for state $m^{\prime}$,

$$
\begin{aligned}
b_{m^{\prime}}= & -\frac{i}{v} \int_{-\infty}^{+\infty} d z \sum_{n}\left\langle\phi_{m^{\prime}}\left|V_{t}\right| \chi_{n}\right\rangle c_{n 1}-\frac{1}{v^{2}} \int_{-\infty}^{+\infty} d z\left\langle\phi_{m^{\prime}}\left|V_{t}\right| \chi_{n}\right\rangle \sum_{n^{\prime}, n^{\prime \prime}, m} U_{n n^{\prime}}(z,-\infty) \int_{-\infty}^{+\infty} d z^{\prime} U_{n^{\prime} n^{\prime \prime}}^{\dagger}\left(z^{\prime},-\infty\right) d_{n^{\prime \prime} m}\left(z^{\prime}\right) b_{m} \\
& -\frac{i}{v} \int_{-\infty}^{+\infty} d z \sum_{m}\left\langle\phi_{m^{\prime}}\left|V_{t}\right| \phi_{m}\right\rangle \beta_{m}(z) b_{m} .
\end{aligned}
$$

Finally, the scattering amplitudes are obtained by solving a set of equations of Eq. (2.13).

Before solving Eq. (2.13), we have to evaluate the excitation matrix elements, charge transfer, and overlap matrix elements. A three-dimensional integral (2.14) has been used to evaluate the excitation matrix elements

$$
\begin{aligned}
\int_{-\infty}^{+\infty} & \frac{e^{-f|\mathbf{r}-\mathbf{R}|}}{|\mathbf{r}-\mathbf{R}|} e^{-i \mathbf{v} \cdot \mathbf{r}} \frac{e^{-\alpha r}}{r} d \mathbf{r} \\
\quad= & \frac{2}{\pi} \frac{1}{\left(v^{2}+\alpha^{2}-f^{2}-2 i \mathbf{v} \cdot \nabla_{\mathbf{R}}\right) R}\left[e^{-f R}-e^{-\alpha r-i \mathbf{v} \cdot \mathbf{R}}\right] .
\end{aligned}
$$

To calculate the excitation matrix elements of $s-p$ and $p-p$, a factor of $z, x, x z$, etc. has to be introduced in the integrand by differentiating with respect to $-i v_{z},-i v_{x}$, etc. of Eq. (2.14). To calculate charge transfer and overlap matrix elements the three-dimensional integral has been reduced to one-dimensional integral in Eq. (2.15) using a Feynman integral technique,

$$
\begin{aligned}
\int_{-\infty}^{+\infty} \frac{e^{-f|\mathbf{r}-\mathbf{R}|}}{|\mathbf{r}-\mathbf{R}|} e^{-\alpha r} \frac{e^{-i \mathbf{v} \cdot \mathbf{r}}}{r} d \mathbf{r} \\
=4 \pi \int_{0}^{1} e^{(i(x-1) \mathbf{v} \cdot \mathbf{R}} \frac{e^{-\sqrt{M} R}}{2 \sqrt{M}} d x,
\end{aligned}
$$

where $\mathbf{v}$ is the velocity of the incoming proton and $f$ and $\alpha$ are the coefficients related with the initial and final states. $M=v^{2} x(1-x)+\left(f^{2}-\alpha^{2}\right) x+\alpha^{2}$. To calculate charge-transfer matrix elements of $s-p$ and $p-p$, a differentiation with respect to $-f,-\alpha,-i v_{z}$, or $-i v_{x}$ has to be taken to introduce $z, x, x z$, etc. in the integrand. The one-dimensional integral of Eq. (2.15) is finally evaluated using the Gaussian quadrature method.

\section{RESULTS}

The results of charge transfer calculated in OHCE are plotted in Figs. 2-4. The data of Cheshire and Gallaher [16] and Shakeshaft [19] and experimental results [27] are also given in the figures. The total charge-transfer sec-

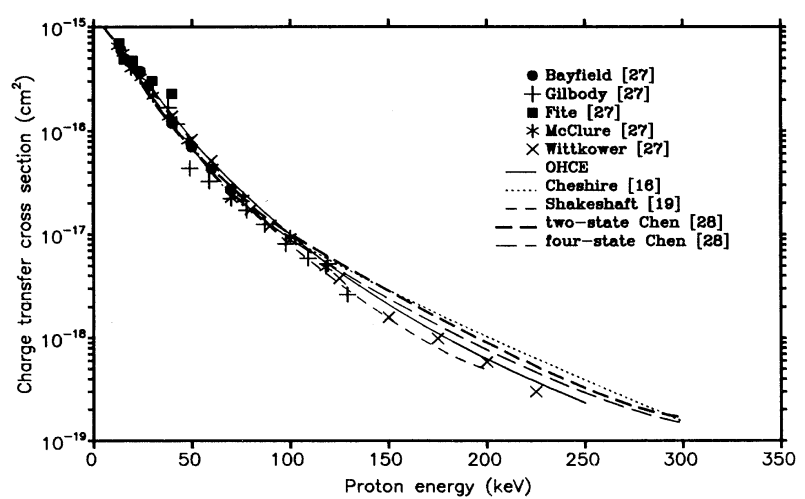

FIG. 2. Charge-transfer cross sections of $1 s$ - $1 s$ in protonhydrogen scattering. Solid line, OHCE calculation; dotted line, four-state Cheshire; dashed line, Shakeshaft; long thick-dashed line, two-state Chen; long thin-dashed line, four-state Chen. Experimental data are measured by Bayfield, Gilbody, Fite, McClure, and Wittkower. 


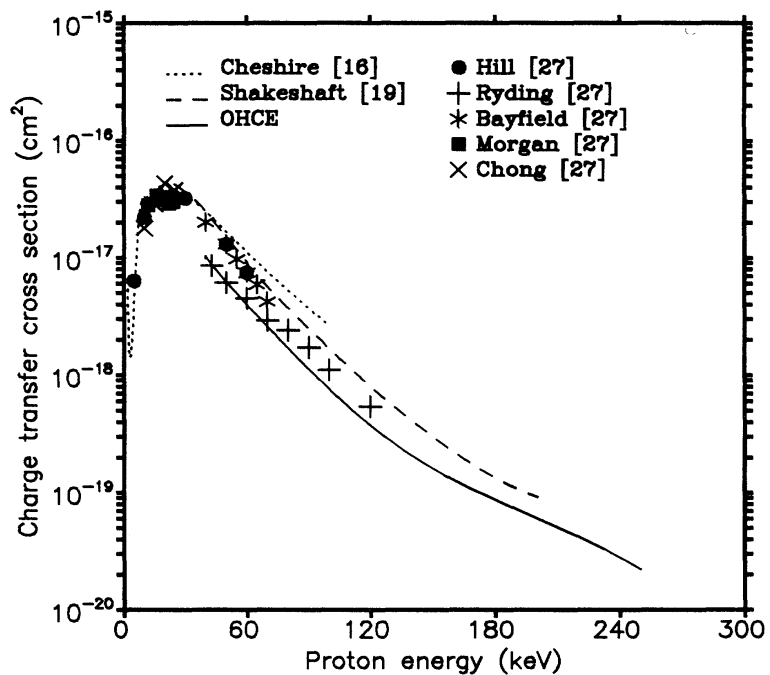

FIG. 3. Charge-transfer cross sections of $1 s-2 s$ in protonhydrogen scattering. Indications of the curves are the same as those in Fig. 2. Experimental results are taken from Hill, Ryding, Bayfield, Morgan, and Chong.

tions were measured by Bayfield, Gilbody, Fite, McClure, and Wittkower. The ratio of Shakeshaft's total to the $1 \mathrm{~s}$ state transfer is used to obtain the $1 s-1 s$ charge-transfer cross sections. The data of $1 s-2 s$ charge transfer were taken from the results of the experiments of Hill, Ryding, Morgan, and Chong. The cross sections of $1 s-2 p$ exchange were the results of Kondow, Morgan, and Stebbings. All the experimental data are listed in Ref. [27].

In Fig. 2 the charge-transfer cross sections from the ground state to the projectile $1 s$ state are plotted. The charge transfer to the $1 s$ state on projectile is large at low energies because the change of binding energy is zero.

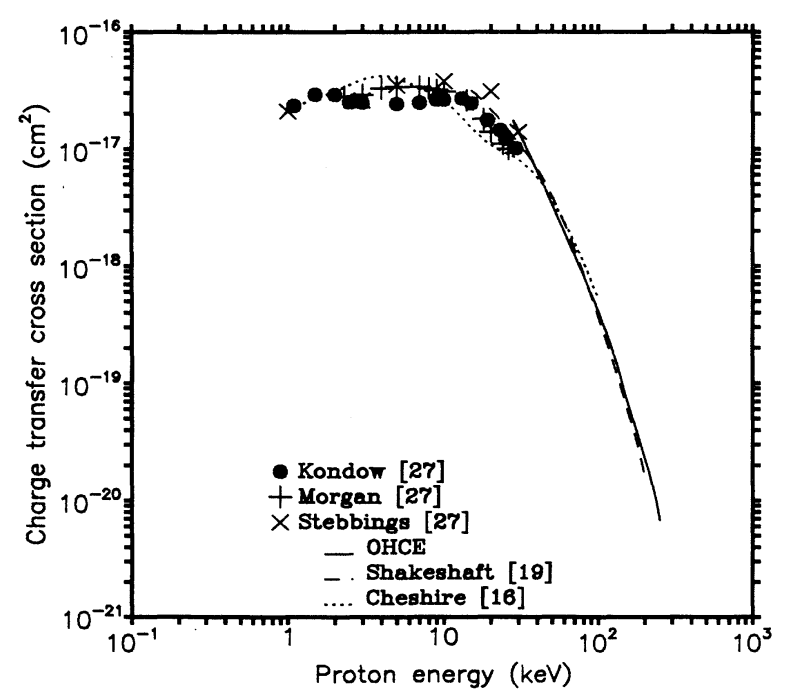

FIG. 4. Charge-transfer cross sections of $1 s-2 p$ in protonhydrogen collision. Curves are indicated in the same manner as in Fig. 2. Experimental data are given by Kondow, Morgan, and Stebbings.
However, as proton energy increases the cross section drops rapidly. This is caused by the difficulty that the electron has in acquiring the necessary kinetic energy. The curves designated as two-state Chen [28] and fourstate Chen [28] in Fig. 2 are calculated in present work using our matrix elements. By comparison of these curves the $1 s-1 s$ charge transfer appears to be converged by including only the $1 s$ states in the wave expansion when energies are low. This is because at low energies the large impact parameters determine the magnitude of the charge-exchange cross sections. At these impact parameters the probabilities for excitations to other states are small. When the energies increase the probabilities for excitations are comparable to the charge transfer to the $1 s$ state. Therefore, to get convergence more states are needed in the expansions of wave function. By including nine radial $s$ states, nine radial $p$ states for the target the OHCE method gives better results than those of twostate Chen and four-state Chen curves in our calculation. It shows that the pseudostates in high excited energies region also contribute to the charge transfer to the $1 s$ state on projectile. The agreement of $\mathrm{OHCE}$ and experiments is excellent in the entire energies range of 30 to $250 \mathrm{keV}$. At low energies our data agree with the results obtained in previous work of partial wave expansion method [23]. When proton energy is above $100 \mathrm{keV}$ the charge-transfer matrix elements calculated in partial wave expansion method gives a significant error. The Feynman integral technique is satisfactorily accurate.

The charge-transfer cross sections to the $2 s$ state are displayed in Fig. 3. The charge-transfer curve exhibits a maximum near $30 \mathrm{keV}$. This is the energy where the proton velocity equals the electron velocity in $1 s$ state. The OHCE curve fits the experimental data of Ryding fairly well. However, our results are consistently lower than the data of Cheshire and Shakeshaft. This may be due to the fact that the $2 s$ state was represented only approximately in our wave expansions.

Figure 4 gives the $2 p$ exchange cross sections. Shown also in the figure are the experimental data of Kondow, Morgan, and Stebbings and the theoretical results of the calculation by Cheshire and Shakeshaft. Our results can fit the experimental data at $30 \mathrm{keV}$. For the proton energies above $30 \mathrm{keV}$, the OHCE curve is almost the same as the curve of Cheshire and Shakeshaft.

\section{CONCLUSION}

In this paper the one-and-a-half-centered expansion has been established as a feasible method by which to calculate charge-transfer cross sections for proton-hydrogen scattering. A code has been developed to allow all $s$ and $p$ states on the projectile to be occupied by the electron.

The Feynman integral technique has been used as an alternative method to evaluate the charge-transfer matrix elements. The one-dimensional integrals have been derived and evaluated using a Gaussian quadrature method. In practical calculations these charge-transfer matrix elements can be used up to several hundred $\mathrm{keV}$ of the proton energy.

A smooth $\beta_{m}(z)$ function which is calculated from the 
SCE has been successfully used to modulate the chargetransfer amplitudes. The $\beta_{m}(z)$ function increases gradually from 0 at $-\infty$ to 1 at $\infty$. It offers an improvement over the step function used in previous work.

The charge transfer to the $1 s$ state has been calculated and gives good agreement with the experimental data. It proves the existence of the contribution of the high excited energy level on target to the $1 s$ charge transfer when proton energies are high. The proton energy ranges have been extended from $100 \mathrm{keV}$ in previous work to 250 $\mathrm{keV}$. This is possible as the present matrix elements are much more accurate than those evaluated using a truncated partial wave expansion, especially in the highenergy region. The charge-transfer cross sections to the $2 p$ state fit the experimental data at $30 \mathrm{keV}$ and are al- most the same as those calculated using the four-state TCE method by Cheshire in the energy range from 30 to $250 \mathrm{keV}$. Charge transfer to the $2 s$ state is also in fairly good agreement with Ryding's experimental data. The computing time is competitive with other methods, but a large memory space is needed.

\section{ACKNOWLEDGMENTS}

This work was supported by the National Science Foundation under Grant No. PHY-9009717. The authors would like to thank Professor A. L. Ford for his very helpful discussions and Professor A. Z. Msezane for his support of this work at Clark Atlanta University.
*Present address: Center for Theoretical Studies of Physical Systems, Clark Atlanta University, Atlanta, GA 30314.

[1] J. R. Oppenheimer, Phys. Rev. 31, 349 (1928).

[2] J. D. Jackson and H. Schiff, Phys. Rev. 89, 359 (1953).

[3] R. A. Mapleton, Phys. Rev. 126, 1477 (1962).

[4] K. Omidvar, Phys. Rev. 153, 121 (1967).

[5] D. R. Bates, Proc. R. Soc. London Ser. A 247, 294 (1958).

[6] M. B. McElroy, Proc. R. Soc. London Ser. A 272, 542 (1963).

[7] T. Pradhan, Phys. Rev. 105, 1250 (1957).

[8] I. M. Cheshire, Proc. Phys. Soc. 82, 113 (1963).

[9] J. P. Coleman and M. R. C. McDowell, Proc. Phys. Soc. 85, 1097 (1965).

[10] D. R. Bates, H. S. W. Massey, and R. A. Smith, Proc. R. Soc. London Ser. A 142, 142 (1933).

[11] D. R. Bates and R. McCarroll, Proc. R. Soc. London Ser. A 245, 175 (1958).

[12] A. F. Ferguson, Proc. R. Soc. London Ser. A 264, 540 (1961).

[13] R. McCarroll, Proc. R. Soc. London Ser. A 264, 547 (1961).

[14] S. E. Lovell and M. B. McElroy, Proc. R. Soc. London Ser. A 283, 100 (1965).

[15] L. Wilets and D. F. Gallaher, Phys. Rev. 147, 13 (1966).
[16] I. M. Cheshire and D. F. Gallaher, J. Phys. B 3, 813 (1970).

[17] D. Rapp, D. Dinwiddie, and D. Storm, Phys. Rev. 5, 1290 (1972).

[18] D. Rapp and D. Dinwiddie, J. Chem. Phys. 57, 4919 (1972).

[19] Robin Shakeshaft, Phys. Rev. A 14, 1626 (1976).

[20] D. R. Bates and A. H. Boyd, Proc. Phys. Soc. 79, 710 (1962).

[21] I. M. Cheshire, Proc. Phys. Soc. 92, 862 (1967).

[22] D. R. Bates, Proc. R. Soc. London Ser. A 245, 289 (1958).

[23] J. F. Reading, A. L. Ford, and R. L. Becker, J. Phys. B 14, 1995 (1981).

[24] A. L. Ford, J. F. Reading, and R. L. Becker, J. Phys. B 15, 3257 (1982).

[25] J. F. Reading, A. L. Ford, and R. L. Becker, IEEE Trans. Nucl. Sci. NS-28, 1089 (1981).

[26] G. L. Swafford, Ph.D. thesis, Texas A\&M University, 1978 (unpublished).

[27] H. Tawara, T. Kato, and Y. Nakar, At. Data Nucl. Data Tables 32, 235 (1985).

[28] Zhifan Chen, Ph.D. thesis, Texas A\&M University, 1991 (unpublished). 